\title{
A change propagating model transformation language
}

Laurence Tratt, Bournemouth University, Poole, Dorset, BH12 5BB, United Kingdom.

Model transformations are a key component in Model Driven Development, but most approaches only allow 'one shot' transformations to be expressed. Change propagating model transformations are those which can make suitable updates to models after an initial transformation. In this paper I outline the challenges presented by change propagating model transformations, before presenting a new change propagating model transformation approach.

\section{INTRODUCTION}

As the use of models - often, but not exclusively, in the form of UML models - in software development increases, the need for model transformations has increased $[3,10,14]$. A simple definition of a model transformation is that it is a program which mutates one model into another; in other words, something akin to a programming language compiler. Of course, if this simple description accurately described model transformations, then General Purpose Languages (GPLs) would suffice to express model transformations. In practise, model transformations present a number of problems which imply that dedicated approaches are required [18].

In recent times, many different model transformation approaches have been proposed (see e.g. [9, 7] for overviews of different approaches). Virtually all of these approaches share in common that they are stateless; that is, after an initial transformation, the only possible action is to rerun the transformation from scratch creating an entirely new target model regardless of whether a target model has previously been created.

In contrast to stateless transformations are change propagating transformations. Alanen and Porres provide a useful overview of change propagating transformations, which also explains some of the categories of changes that can be propagated [1]. A possible scenario is when tools which specialize in different aspects of modelling can be used together throughout the development life cycle e.g. a UML modelling tool $U T$ and a Java modelling tool JT. In such a scenario, a model is not just transformed between different tools once, but may be edited multiple times in each tool. For example, an initial model may be created in UT, transformed and subsequently edited in $J T$, before high-level architectural changes are applied in UT which one expects to see reflected in JT. A similar, although more linear, scenario involving

Cite this article as follows: Laurence Tratt: A change propagating model transformation language, in Journal of Object Technology, vol. 7, no. 3, March-April 2008, pages 107-126, http://www.jot.fm/issues/issues_2008_3/article4 
incremental model development is explained in Becker et. al [2]. The general idea underlying such scenarios is of allowing the user to leverage different tools specialities at varying points in the development life cycle. In previous work I outlined some of the other possible uses of change propagating transformations [18].

Currently very little focus has been given to the challenging problem of change propagating model transformations. To my knowledge, only three approaches tackle this problem in any way: BOTL [4], Johann and Egyed's approach [12], and XMOF [6]. All three approach the problem in very different ways, and with varying degrees of success. From this one can infer that the fundamentals of change propagating model transformations have yet to be identified.

In this paper I present a new approach to change propagating model transformations called PMT. PMT is a follow up to my work motivating the need for change propagating transformations [18], and the unidirectional stateless model transformation MT [20]. This paper is a condensed version of the technical report [19] which contains much more detail than the space constraints of journal publication allow. The aim of PMT has not been to present a definitive change propagating model transformation approach; rather the aim has been to explore the practical challenges of implementing a system, and to discover the high-level strategies and design decisions relevant to change propagation. I believe that PMT offers a number of insights into the practicalities of change propagating model transformations, and serves as a solid base from which to build subsequent systems.

This paper is structured as follows. I first give a brief definition of change propagating model transformations, outlining some generic challenges for any change propagating model transformation approach. I then describe by example PMT itself. I conclude by separating out the parts of PMT that are fundamental to change propagation, thus providing a useful basis for future study of change propagating model transformations.

\section{CHANGE PROPAGATING MODEL TRANSFORMATIONS}

A unidirectional change propagating model transformation is considered to consist of a source model and a target model. Typically before the transformation is first run, the source model is populated with one or more model elements, and the target model is empty. After running the transformation for the first time, the target model is populated with model elements. After this the user may then manually edit both the source and target models, adding, removing, and altering elements. At some point the user reruns the transformation and any changes made to the source model should be non-destructively propagated to the target model. The use of the term 'non-destructive' is deliberate: in propagating changes from the source model, any manual updates to the target model must be preserved.

This simple description of change propagation does not include a number of the decisions and challenges that any particular approach must consider. I believe that 
the following list (though not exhaustive) contains many of the major decisions and challenges:

Checking or updating propagation. At a minimum, a change propagation approach may tell the user where changes need to be made to the target model to make it conformant; at the other extreme an approach may forcibly update the target model without informing the user of which changes will be made.

Manual or automatic change propagation. [21] outlines a framework where changes to the source model are extract as change deltas which are then passed to delta transformations which apply the change delta to the target model. I term this manual change propagation since a potentially unlimited number of delta transformations need to be manually written to propagate changes. In contrast, automatic change propagation is when an approach can propagate arbitrary changes without manual help. Some systems may use a mix of both approaches [5].

Batch or immediate propagation. Batch change propagation takes a number of changes from the source model and propagates them to the target model only when explicitly requested to do so by the user. The concept of immediate change propagating transformations is defined in [6]. An immediate change propagating transformation propagates changes to the target model as soon as the source model is changed. Unlike a batch mode change propagating transformation, which implicitly propagates multiple changes when run, an immediate mode change propagating transformation propagates small changes, which can be viewed as being semi-atomic.

Relating source and target model elements. Every change propagating approach requires a mechanism that relates (or somehow distinguishes), the specific target model elements created by a given rule relative to specific source elements. The distinguishing of elements is vital to ensure that target elements are modified, created or removed correctly during change propagation.

Detecting elements for removal. Removing source model elements should lead to the relevant elements in the target model also being removed (provided such removals preserve the property of non-destructivity of manual changes).

Checking correctness of propagation and conflict resolution. Some changes made to a source model may not be able to be propagated successfully to the target model. For example, when propagating an element newly added to the source model, a conflict may arise with an element already present in the target model.

A lengthier discussion of these items can be found in [19]. 


\section{PMT}

PMT is a new approach to change propagating transformations. In terms of the design decisions enumerated in section 2, PMT can be said to be a fully automatic, batch, change propagation approach, which relates source and target elements by their identifiers, and which has user controllable correctness checking built in. The details of this broad overview will be filled in as this paper progresses. PMT is, at a low level, a fork of the MT model transformation language [20]; many of the mundane details of MT apply equally to PMT and are not repeated here. As with MT, PMT is defined as a DSL within the Converge language [17]. However whereas MT is in essence an imperative model transformation language, PMT is in essence declarative.

PMT is interesting for several reasons. As well as being one of the first published change propagating model transformation approaches, it is the first with a publicly available implementation. It is also perhaps unusual in that while it is overall a declarative model transformation approach - where rules define a relationship between source and target model elements - it is housed within an imperative language, and rules can themselves contain arbitrary chunks of imperative code. Thus in PMT the dividing line between imperative and declarative - always somewhat contentious terms - is extremely blurred.

\section{An overview of PMT}

A PMT transformation has a name and consists of one or more rules, the ordering of which is significant. Rules are declarations, implemented as functions, which define the source elements they match against, and the target elements that should exist in the target model given a successful match. Rules are comprised of: a source matching clause containing one or more source patterns; an optional when clause on the source matching clause; a target producing clause consisting of one or more expressions; and an optional where clause for the target production clause.

A PMT transformation takes in one or more source elements, which are referred to as the root set of source elements. The transformation then attempts to transform each element in the root set of source elements using one of the transformations rules, which are tried in the order they are defined. If a given element does not cause any rule to execute then an exception is raised and the transformation is aborted. The general form of a PMT transformation is as follows:

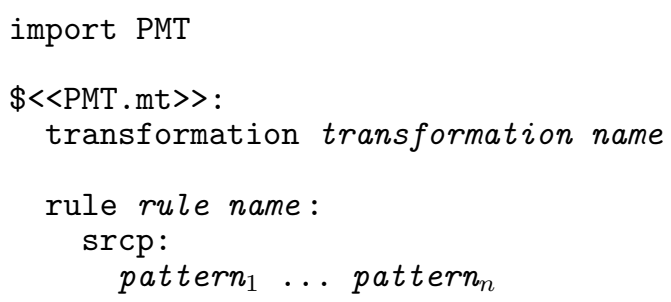




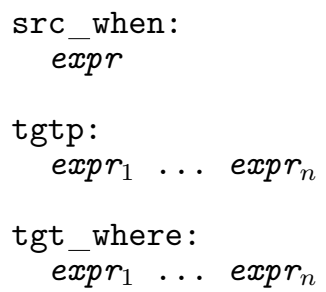

The import statement is a normal expression in the Converge language and imports the MT module. DSL blocks are introduced by $\$<<\ldots>>-$ in this example an PMT model transformation DSL block. Since Converge is an indentation based language, all code indented from the $\$<<\mathrm{MT} . \mathrm{mt}\rangle>$ line is part of the DSL block; note that code preceding $\$<<\mathrm{MT} . \mathrm{mt}>>$ is normal Converge code, as is any code following the DSL block. As this example shows, a Converge DSL can conform to an arbitrary grammar. A DSL block is translated into a Converge abstract syntax tree using Converge's compile-time meta-programming facilities. Arbitrary Converge code can be embedded inside the DSL block itself (see [17, 20] for more details on these mechanisms).

The srcp and srcp_when clauses are collectively said to form the source clauses; similarly the tgtp and tgtp_when clauses are collectively said to form the target clauses. Transformation rules contain normal Converge code in expressions; such expressions can reference variables outside of the model transformation DSL fragment. Users can thus call arbitrary Converge code, allowing them a means to extend the model transformation approach as necessary. This is an important aspect of PMT since it allows users to use normal Converge functions arbitrarily, and without penalty; this stands in contrast to many model transformation approaches which provide a 'closed world' which can not easily be extended.

\section{Pattern language}

PMT's pattern language is a super-set of that found in the QVT-Partners approach. PMT defines a number of pattern expressions: model element patterns, set patterns, variable bindings, and normal Converge expressions. Model element patterns are of the form (Class, <self_var >) [slot name == pattern]. A model element pattern matches against a model element of type Class, and then checks each slot comparison slot name against a pattern pattern. If the type check, or any of the slot comparisons, fails then the entire model element pattern fails. In general, any of the standard Converge comparison operators (e.g. ==, >= etc.) can be used in slot comparisons, and the same slot name may be involved in multiple comparisons in any given model element expression. If the type of the model element pattern, or any slot comparisons, fail then the model element pattern itself fails. Set patterns are directly analogous to those found in functional languages such as Haskell. Variable bindings $\langle v\rangle$ intuitively mean 'match anything and bind to $v$ '. If the same variable binding appears more than once in the same scope, all instances of that variable name must match against equivalent objects (the QVT-Partners approach does not 
define its own notion of equality, instead inheriting it from the MOF [15]). Converge expressions, when used as patterns, match only against a model element which compares equal to the evaluated Converge expression. If a model element expression successfully matches against a model element, then the model element is bound to the optional self variable self_var.

As a trivial example of a model element pattern, assuming an appropriate metamodel, the following example will match successfully against a Dog model element whose owner is not Fred, binding the matching Dog element to the variable $d$ and its name to $\mathrm{n}$ :

$($ Dog, $\langle\mathrm{d}\rangle)[$ name $==\langle\mathrm{n}\rangle$, owner $==($ Person $)$ [name $!=$ "Fred"]

\section{Execution strategy}

PMT can execute as both as both a full and semi-conservative updating propagation approach, and the two modes can be arbitrarily intertwined. When PMT is executing in fully conservative mode, it forcibly propagates only those changes which do not interfere with any existing target model elements; in semi-conservative mode it attempts to forcibly propagate all changes. In both cases, the overall execution strategy is the same: PMT executes, attempting to make the source and target models conformant with respect to each other and the transformation.

The use of the phrase 'make the source and target models conformant with respect to each other and the transformation' is deliberate: a PMT transformation is essentially a constraint, and for any given transformation and a particular source model there may potentially be many conformant target models (and vice versa). Making a target model conformant may require elements being added, altered, or removed from the target model. The way in which the relationship between source and target elements is specified, and the process by which the update of the target model occurs are the two defining aspects of PMT.

\section{A PMT transformation's stages}

The stages of a PMT transformation are as follows:

1. Take a source model, and an empty target model and transform the source model. This stage - if taken in isolation and viewed as a black box - is simply that of a standard unidirectional model transformation. After the transformation has executed, the source and target models, together with tracing information created, are stored in some fashion.

2. The user may make arbitrary changes to both the source and target models, independent from one another. 
3. The user then requests that the changes they have made to the source model are propagated non-destructively to the target model. The transformation is reinitialized with the updated source and target models, and the tracing information from the previous execution. The execution of the transformation then propagates changes from the source model to the target model. After the transformation has executed, the source and target models, together with the new tracing information created are once again stored.

At this point, the sequence moves back to stage 2 .

\section{EXAMPLE}

The example I use in this paper is a version of the standard 'class to relational model' transformation [16], reduced in complexity in the interests of brevity. The 'Simple UML' meta-model is shown in figure 1, and the relational database meta-model in figure 2. Essentially each class is translated to a table. One or more of a classes attributes must be marked as being part of its primary key; attributes either have a primitive (e.g. integer, string) type or refer to another class for their type; if an attributes' type is that of another class, the primary key of that class is used as a foreign key (recursively, and possibly spanning multiple columns).

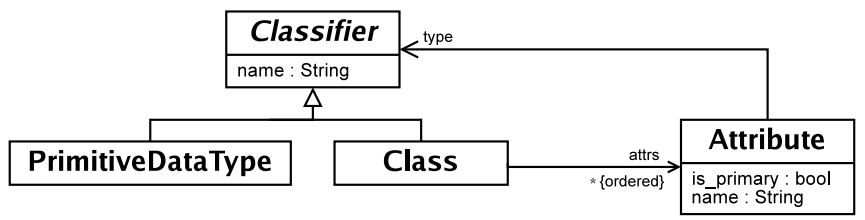

Figure 1: Extended 'Simple UML' meta-model.

\begin{tabular}{|c|c|c|}
\hline Table & 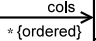 & Column \\
\hline name : String & $\stackrel{\text { pkey }}{\longrightarrow}$ & $\begin{array}{l}\text { type : String } \\
\text { name: String }\end{array}$ \\
\hline
\end{tabular}

Figure 2: Extended relational database meta-model.

The transformation itself is as follows:

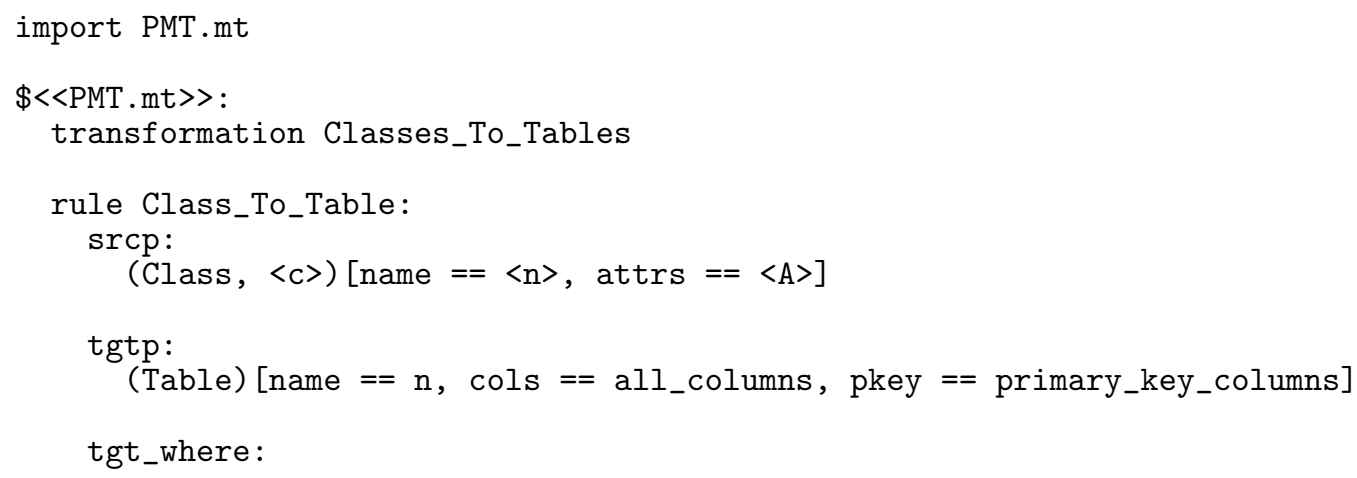




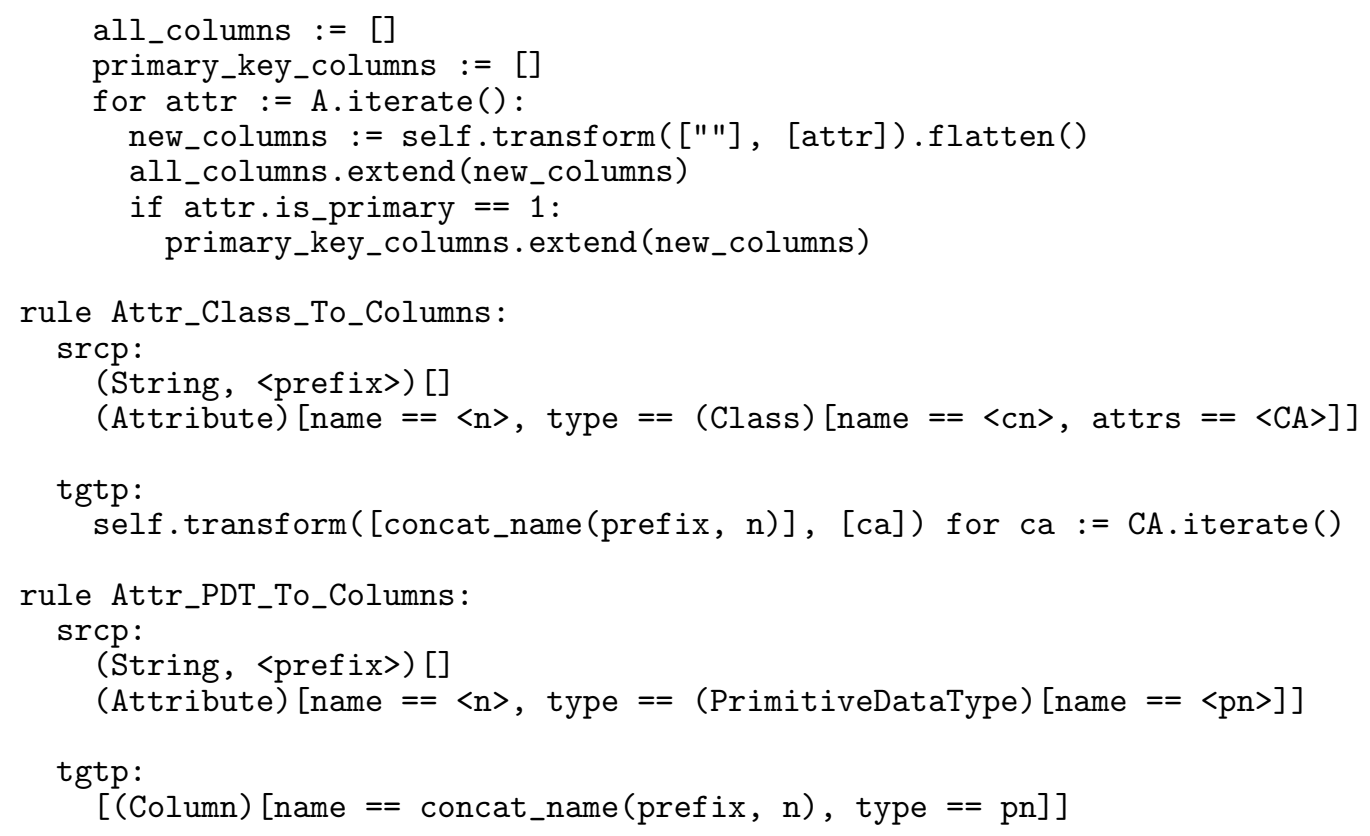

The overall structure of this transformation is hopefully relatively straightforward even if some of the finer details are not. The Class_To_Table rule ensures that each class transformed into a table in the target model. Attr_PDT_To_Columns transforms an attribute with a primitive type into a single column. Attr_Class_To_Columns transforms an attribute whose type is another class into one or more columns, building up the prefix of the eventual column(s) name. concat_name ( $p$, $\mathrm{n}$ ) is a normal Converge function which concatenates the string $\mathrm{p}$ with $\mathrm{n}$ (separating the two by an underscore) if $\mathrm{p}$ is empty, otherwise returning $\mathrm{n}$.

Three features in this transformation need extra explanation in the context of this paper. First, the self variable in Converge code is analogous to this in Java - PMT transformations are in fact translated to a Converge class, and one can thus access specific rules and so on via the self variable. Second, the transform function used throughout the transformation is also present in every PMT transformation. It takes an element(s) in, and successively tries every transformation rule in the transformation using the arguments passed to it, attempting to find one which executes given the element(s) as input. If no rule executes, the transform function raises an error. The transform function is used internally by PMT to transform each element in the root set but, as in this example, may be called at will by the user. Third the for keyword in target pattern of the Attr_Class_To_Columns rule is used to generate multiple target elements; essentially for each time time that the expression on the right hand side of the for expression evaluates successfully (using the standard Converge semantics), a new target element will be created. 


\section{Example execution}

Figures 3 and 4 shows the automatic visualization of a source model and resulting target model from running the transformation of the previous section, in UML object diagram format. The relationship between source and target is simple at this stage; the main detail to note is that source models are always presented in blue, and target models in green.

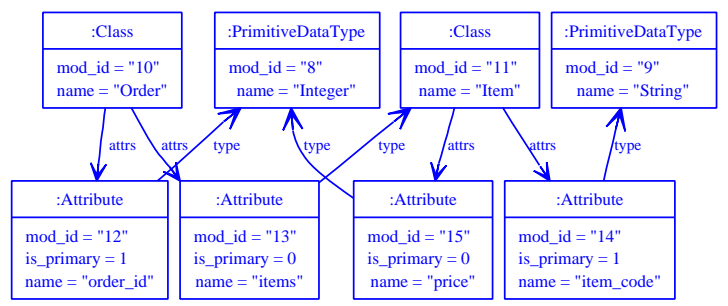

Figure 3: Source 'Simple UML' model.

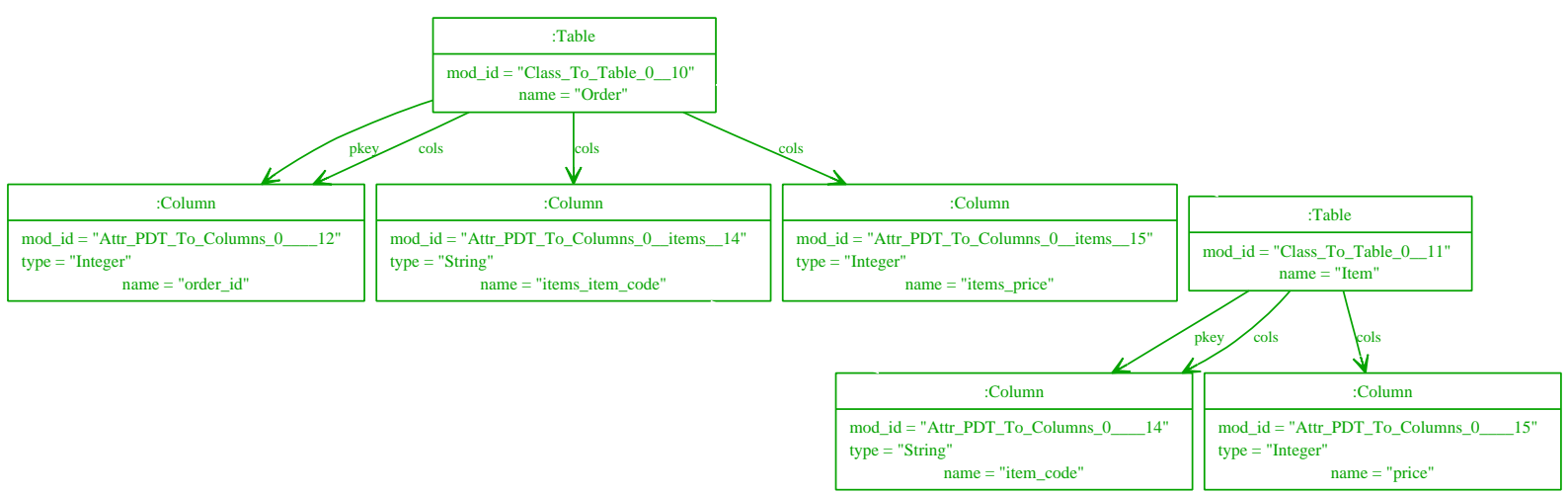

Figure 4: Target relational database model.

At this point, we make the following changes to the source and target models: we rename the Item class to Stock_Item; remove the items attribute from Order, replacing it with a Order_Item class that can track changing stock prices over time; and we change the name of the Order table in the target model to Cust_Order. The source model at this point looks as in updated source simple UML model.

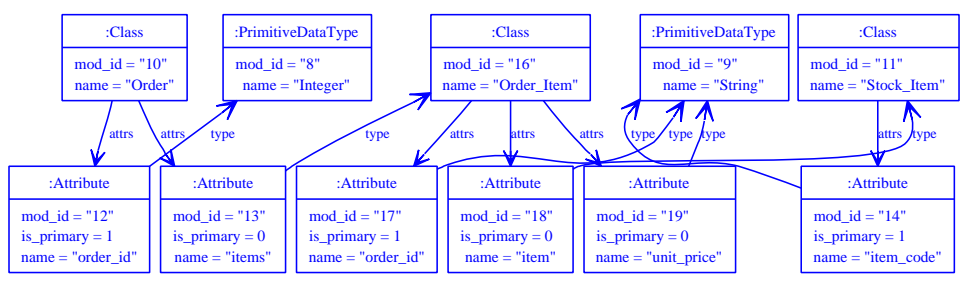

Figure 5: Updated source 'Simple UML' model.

After re-running the transformation, the target model looks as in 6 . The obvious difference in figure 6 is the large number of model elements in red, which denote 
conflicts. The reason for these red elements is that the transformation defined in section 4 is a fully conservative transformation. When the transformation is run (initially or subsequently), it attempts to transform source elements; those which it can transform without interfering with pre-existing target model elements are transformed (hence the appearance in figure 6 of the new Order_Item table). Those source elements which would interfere with pre-existing target model elements are labelled in red.

In the following subsections I go into more detail about conflicts, how PMT relates source and target model elements, and how PMT transformations can be turned from semi-checking into updating change propagation approach.

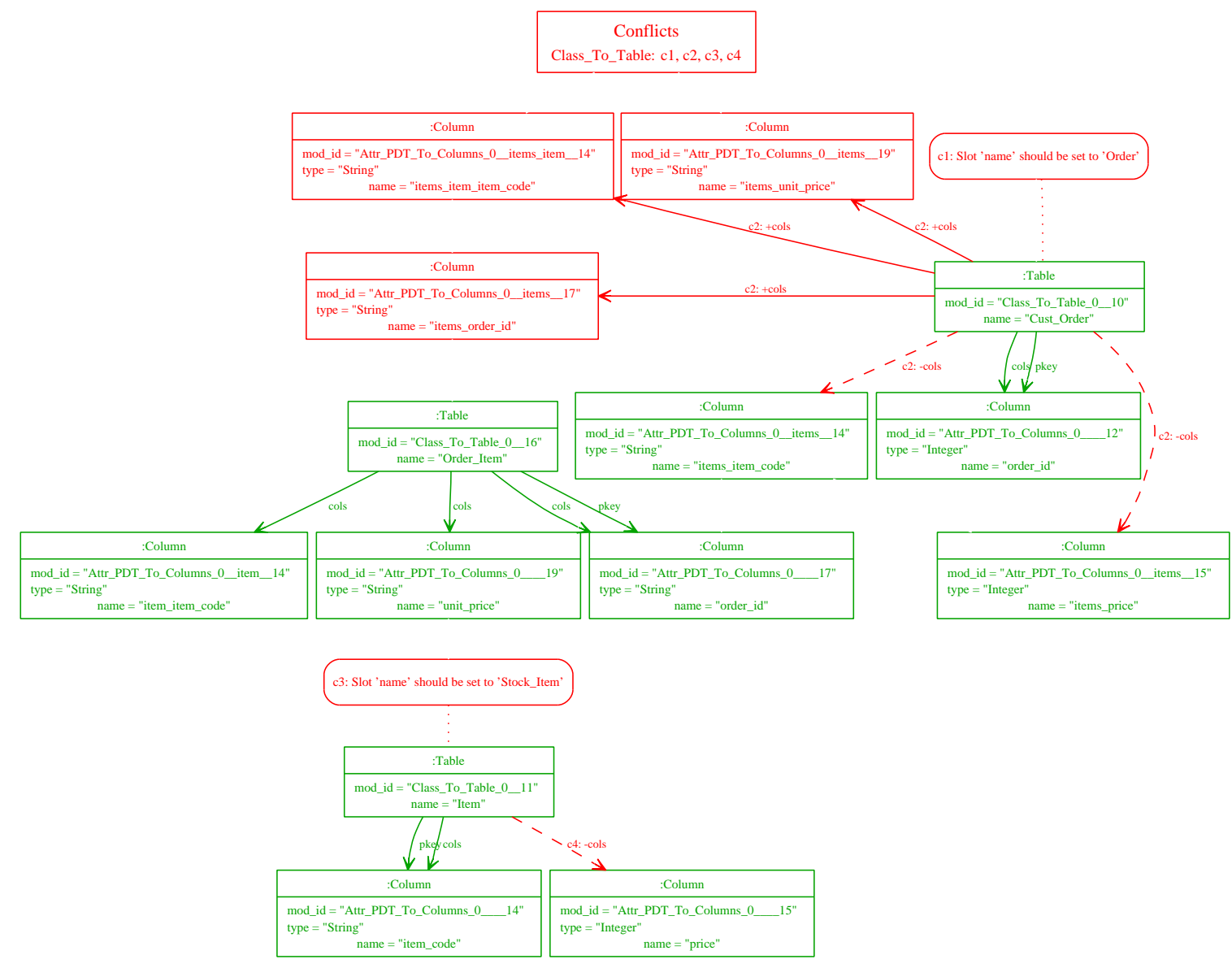

Figure 6: Updated target relational database model.

\section{Conflicts}

Figure 6 shows a number of model elements in red. Elements and links shown with solid red lines are those which need to be added to the target model in order to make it conformant; elements and links shown with a dashed red line to be removed. Boxes with rounded corners are informational (saying that a particular slot in an 
element needs to be changed and so on). Collectively these conflicts constitute what can be thought of as a visual 'diff' [11]; if all of the changes indicated are made, then the source and target models are conformant with each other relevant to the transformation.

There is also other interesting, and sometimes important, information that can be gleaned from figure 6 . At the top of the figure is a legend box which records which rules led to conflicts being generated; it is deliberately similar to the visualization of traces in the MT model transformation language [20]. In the case of figure 6 , one can see that four separate executions (labelled c1 to c4) of the Class_To_Table rule led to conflicts. Each conflict in the diagram itself is labelled with one of these executions; as can be seen from execution c2, multiple conflicts may result from one execution of one rule. By looking at the execution number on an element or link, one can determine not only which rule led to the conflict, but whether other elements were involved in the same execution of that rule.

One question raised by figure 6 is: why are two cols links from the Cust_Order element in dashed red, but not the elements to which the links point? This is tackled in section 5 .

\section{Relating source and target model elements}

Up until now I have been deliberately vague as to how PMT knows which source and target elements are related to each other. There are three main ways that one can imagine determining this relationship: by key, by tracing information, and by identifier. In this section I briefly outline these mechanisms, before discussing PMTs approach to relating elements.

\section{Element relation mechanisms}

The three main mechanisms for relating elements, and their pros and cons, can be summarised as follows:

Relating elements by key This involves relating elements by a collection of attributes which, collectively, uniquely identify any given element (as in the standard database notion of key) and is advocated by the DSTC QVT approach [8]. Transformations thus only need to know about the relation of source and target element keys; other information in model elements is essentially irrelevant.

There are two problems with this method. The first is that by requiring models to define keys, an extra burden is placed on the user. Although this is often trivial, it can be difficult when elements have no natural key. The second is more fundamental, and relates to the fact that, after the initial transformation, the user can not safely change source or target elements keys since changing a keys value means the transformation may no longer correctly relate elements. 
Relating target elements via tracing information Tracing information relates which source elements led to the creation of particular target elements. As this information is created by PMT already - PMT uses the same tracing creation scheme as [20] - it seems like a good candidate to relate source and target elements. However, as shown in [20], there are various different tracing information creation mechanisms. The success of a change propagation algorithm then depends on factors such as the coverage and granularity of the recorded tracing information. For example, while the default tracing information generated by PMT records which target elements were created by a rule from specific source elements, it does not generate enough information to know which part of the rule created which target element. Such information may be vital for an accurate change propagation algorithm.

There is thus a potential tension between the different uses of tracing information. The type of tracing information desirable for change propagation may be very different from that required by a user to understand transformations on their model. However, assuming that it is suitably detailed, tracing information is sufficient as the sole means of distinguish elements for change propagation.

Distinguishing target elements by identifier A technique that can ultimately be seen as a slight variation on distinguishing target elements by tracing information was outlined by Johann and Egyed in [12], and independently by this author in [18]. When a target element is created it is given an identifier which contains, at a minimum, the concatenated identifiers of all the source elements which led to the creation of the target element. Henceforth I refer to this as the target element identifier. Note that the target element identifier may be in addition to an elements standard identifier, and that conceptually there is no requirement that this new identifier be a single field.

Conceptually this technique does not add any additional power over using tracing information to distinguish elements. Indeed, a simple concatenation of the source elements identifiers means that the target element identifier is merely an alternative way of storing information that can in theory be directly derived from suitably fine-grained tracing information. However extra information can be easily stored in the target element identifier, if required, to allow a transformation to encode information which may not be present in tracing information. This then allows tracing information to be used for other purposes. Furthermore this then means that tracing information need neither have complete coverage, nor be fine-grained; as such, tracing information can be recorded in a fashion which gives it the greatest utility to the user.

\section{PMTs approach to relating elements}

Since it is the approach with the least cons, PMT distinguishes target elements by identifier. In simplified terms, a rule concatenates together the identifiers of the 
source elements it has matched, and then sees if an element with such an identifier already exists in the target model. If no such element exists, PMT creates a new object. However if such an element exists, it is used as the basis for further operations. Creating suitable target element identifiers is thus a significant part of PMT.

Fundamentally target element identifiers need to satisfy two criteria: that they are unique with respect to particular source elements and a particular rule execution; that they can be created deterministically across multiple transformation executions to allow changes to be propagated multiple times. In order to satisfy these two criteria, PMTs approach to creating identifiers is somewhat more sophisticated than has been suggested, or indeed described in previous publications.

From a purely implementation point of view, PMT uses the mod_id field in model elements to store identifiers. As can be seen from e.g. figure 6, this field stores a string. By default each new model element is given a unique string identifier (a number starting from 0 and monotonically increasing when a new element is created), but new model elements can be assigned an arbitrary model identifier.

Concatenating the identifiers of source elements is not sufficient on its own to generate unique target element identifiers, since the same source elements may be used in more than one rule execution. PMT thus also integrates the name of the rule being executed into the target identifier to ensure that target element identifiers are unique. This is sufficient when a rules target clauses contain a single model element expression which executes only once. If a rule has multiple model element expressions in its target clauses, or if a model element expression can execute more than once in a single execution of a rule, then a single target element identifier might result in multiple target elements being created with the same identifier. In order to ensure uniqueness, each rule execution keeps a counter of how many times model element expressions have been executed during the execution. This counter is incorporated into the target element identifier of model element expressions, thus ensuring the uniqueness of the identifiers even when a rule executes more than one model element expression.

The general form of a target element identifier in PMT is thus as follows:

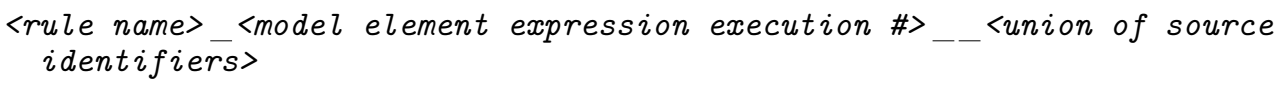

Using this template, one can interpret the identifiers of target elements in figure 6 with respect to the transformation of section 4 .

There is one last potential cause of conflict in the generation of target model identifiers: executing the same rule with the same source elements more than once could lead to conflicting target identifiers being generated. To avoid this possibility, PMT rules keep a cache of source elements that have already transformed: if a rule matches against the same source elements as it did in a previous execution, then the target elements produced in that previous execution are returned. Note that this design decision is vital to ensure the uniqueness of model element identifiers. 


\section{EXTENDING THE EXAMPLE}

The example presented in section 4 showed a transformation executing in fully conservative mode. In this section I show how PMT can execute in semi-conservative mode, that is PMT attempts to forcibly propagate all changes. In order to do this we have to make changes to the transformation itself. The updated transformation is as follows:

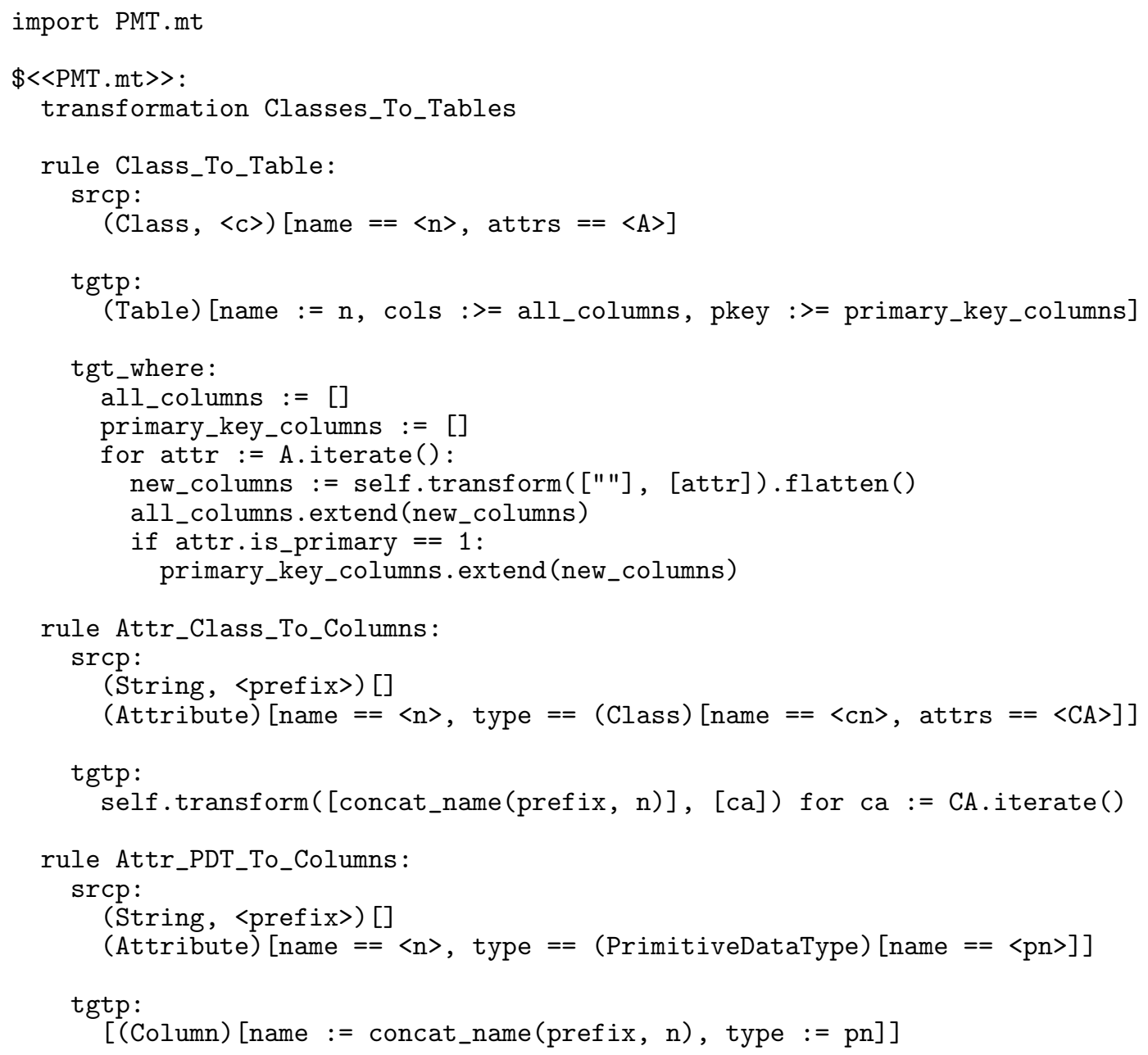

The only changes made to this updated transformation are in the model element expressions in the target clauses of rules. Previously it was possible to think of both source and target clauses containing patterns; indeed PMT's syntax has been deliberately designed to encourage this. However it is now necessary to differentiate the purely matching model element patterns in source clauses from the constructive - and potentially updating - model element expressions in target clauses. Where before each slot comparison (see section 3) in model element expressions was ==, conformance operators such as the following can be used in model element expressions: 


\begin{tabular}{l|l|l} 
Operator & Name & Description \\
\hline$x:=y$ & update & Forcibly sets the value of slot $x$ to $y$ \\
$x:>=y$ & update superset & $\begin{array}{l}\text { The value of slot } x \text { must be a non-strict superset of } \\
y \text { 's value. Any elements in } y \text { not present in } x \text { will } \\
\text { be added to } x . x \text { may contain elements not present } \\
\text { in } y .\end{array}$
\end{tabular}

Running this new transformation on the source model of figure 5, the resultant target model is figure 7 . The most obvious feature of figure 7 is that there are now no elements in red - all the conflicts of figure 6 have been automatically resolved.

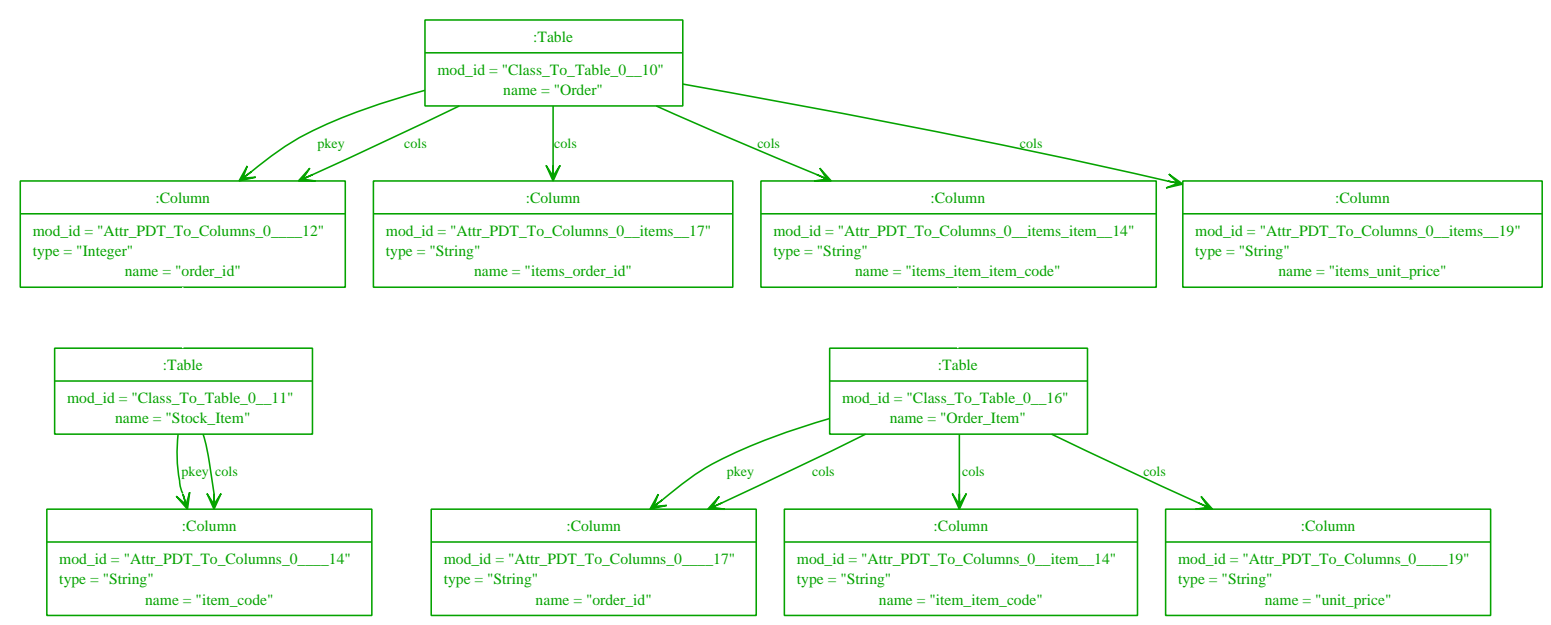

Figure 7: Target model after changes propagated.

The execution strategy for transformations using conformance operators such as those listed above is exactly the same as before. As the transformation executes, it attempts to make target model conformant to the source model and itself by adding or altering elements as needed. If it is unable to make an element conformant, it is possible for conflicts to be raised in the same style as before, although it is rare to hit such cases in practice.

It is easy to assume that the conformance operators above are really just imperative updating expressions in disguise. However conformance operators such as :>= have both updating and constraint properties, that are vital for change propagating transformations. Taking figures 5 and 7 as a basis, let us assume that the user has added an attribute Address of type String to the target class Order, and a column total of type String into the target table Order. Having made these changes, and running the transformation again, an elided version of the target model can be seen in figure 8. Not only has the address attribute change been propagated into the target model, but the manually added total column has been left untouched.

The update superset conformance operator is particularly interesting since it does not imply, or force, the value of the slot in the target element to be directly equal to the value generated by the model expression. Instead, the value of the slot in the 
target element is altered to make sure it contains all the elements that the model expression says it should have; if it has extra elements then those are left intact. I believe this ability to non-destructively propagate changes - i.e. to leave manually modified elements of the target model alone when they do not conflict with the transformation - is vital for real-world change propagating transformations. Note that although PMT contains several other conformance operators, the $:=$ and $:>=$ are by far the most commonly used in practise.

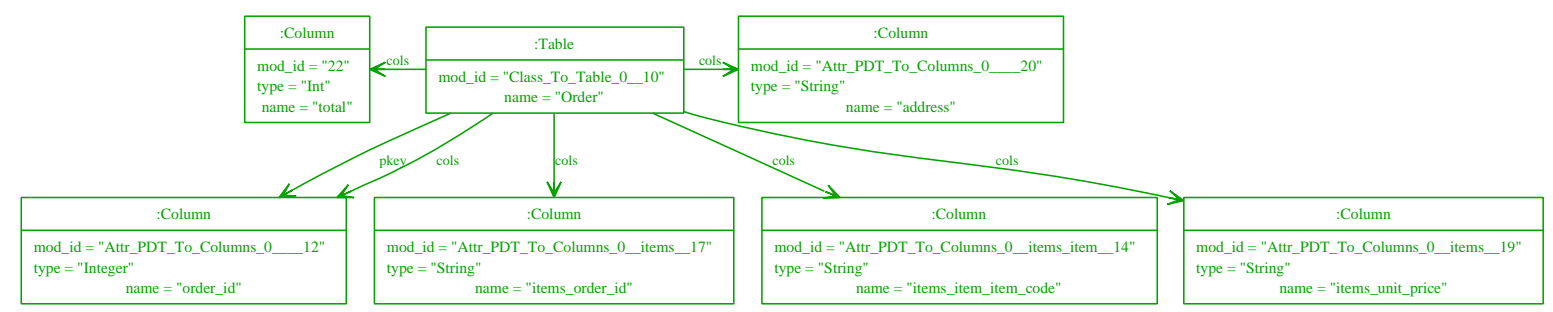

Figure 8: An elided version of a user-modified target model after changes propagated.

One important point that may not be immediately obvious is that transformation writers still need to use careful thought to determine when each should be used. For example, an inexperienced transformation writer may choose to use the update operator in all slot conformances, since this will ensure that all changes made to the source model are propagated automatically to the target model. However if the slot in question contains a set then the users' manual changes made in the target model will be destroyed. In such cases, one would generally expect the transformation writer to use the updating slot conformance operator. In some cases, however, the transformation writer may deliberately wish to ensure that the target model contains the transformed set elements, and nothing else, in which case the update conformance operator is the correct choice. Knowledge of the appropriate situations for each conformance operator is likely to be gathered only through knowledge of the source and target domains, and experience with the change propagating approach.

\section{Removing source elements}

Removing elements from the source model poses a special challenge to change propagation. As explained previously, PMT's approach to propagating changes has always been to propagate new or changed source elements to the target model. If the user removes an element from the source model, one would expect the appropriate element(s) to be removed from the target model on propagation. This requirement may at first appear to be solved by examining all target elements at the end of a transformation execution, and removing those elements which were not created as the direct result of transforming one or more source elements. However this simple solution would also delete any elements manually added to the target model by the user, and as such is clearly not suitable for the use cases PMT is aimed at. The 
critical problem is therefore to distinguish which seemingly superfluous elements in the target model have been manually added by the user, and which are no longer a part of the transformation.

In order to determine which elements can be safely deleted in the target model, PMT utilises tracing information - both that generated by an execution of the transformation, and that generated by its previous execution. After changes have been propagated, a PMT transformation examines every element in the target model, checking whether it is referenced in either or both of the current and previous tracing information. Based on this, PMT draws a conclusion about the origins of the element and whether it is a candidate for removal. The four possibilities for an element are as follows:

\begin{tabular}{|c|c|c|c|}
\hline $\begin{array}{l}\text { In previous } \\
\text { tracing } \\
\text { info.? }\end{array}$ & $\begin{array}{l}\text { In current } \\
\text { tracing } \\
\text { info.? }\end{array}$ & Conclusion & $\begin{array}{l}\text { Candidate } \\
\quad \text { for } \\
\text { removal? }\end{array}$ \\
\hline$\sqrt{ }$ & $\sqrt{ }$ & $\begin{array}{l}\text { Target element previously created by } \\
\text { PMT. }\end{array}$ & $\times$ \\
\hline$x$ & $\sqrt{ }$ & $\begin{array}{l}\text { Target element newly created by } \\
\text { PMT. }\end{array}$ & $x$ \\
\hline$\times$ & $\times$ & $\begin{array}{l}\text { Target element manually added to tar- } \\
\text { get by user. }\end{array}$ & $\times$ \\
\hline$\sqrt{ }$ & $\times$ & $\begin{array}{l}\text { Target element previously created by } \\
\text { PMT; corresponding source element } \\
\text { now deleted. }\end{array}$ & $\sqrt{ }$ \\
\hline
\end{tabular}

Once every element has been examined, PMT performs a garbage collection style 'mark and sweep' [13], using the transformed root set of source elements as the starting point. Any self-contained cycle consisting solely of elements marked as being candidates for removal, is then removed from the target model. The need to identify self-contained cycles of such elements is to prevent the removal of elements cause the target model to become ill-formed. This could occur if elements are removed from the model even though they are referred to by other objects.

\section{RELATED WORK}

Only three model transformation approaches are of potential interest with respect to change propagation: BOTL [4], Johann and Egyed's approach [12], and XMOF [6].

BOTL restricts itself to bijective transformations which is extremely limiting as the majority of real-world transformations fail to satisfy either or both of the injective (commonly known as 'one-to-one') and surjective (commonly know as 'onto') criteria that this requires. 
The XMOF approach is interesting in that it uses full constraint solving to tackle the problem of bidirectional change propagating model transformations. However bidirectional transformations - stateless or change propagating - introduce significant extra complexity into the transformation process. There are two issues with $\mathrm{XMOF}$ that make it rather difficult to use. First, by its very nature, and even with perfectly specified systems, it can take an unbounded amount of time to solve constraints and execute. Second, it places a significant burden on the user to make sure that the constraints they specify completely describe the transformation - if they fail to do this, the resulting transformation is likely to either produce arbitrary results each time it is run, or to run out of memory as it attempts to enumerate all matching values.

Johann and Egyed's approach is the most interesting of the three, and the closest in spirit to PMT, as it tackles unidirectional change propagating model transformations; however it explains only one aspect of its approach in detail, and furthermore is incapable of correctly propagating some important types of changes. Specifically, the approach attempts to optimise propagation so that only updates local to the change are propagated. As shown in [19], local changes sometimes have global effects on the transformation. Because PMT effectively performs a full transformation every time it propagates changes, it can correctly propagate such changes.

\section{LIMITATIONS AND FUTURE WORK}

Although PMT is successful in many ways, as a first-generation change propagating approach, there are several directions in which future work could usefully go. For example, it might be possible to optimise many simple types of changes so that only a subsection of the transformation is rerun; [19] has more some more concrete suggestions along these lines.

Looking in a completely different direction, PMT fundamentally works on the basis of continually updating the target model. An interesting area of research would be for PMT to change to producing diff's against the target model (as in section 4), keeping a complete history of such diffs. This would allow the target model to be manually manipulated by the user, who could choose which aspects of which diffs to include at any given point, in analogous fashion to textual version control systems. By keeping a history of diffs, PMT might also be able to make more intelligent decisions about element deletion by taking into an account an elements entire history.

\section{CONCLUSIONS}

In this paper I have presented the PMT change propagating model transformation language. I explained in detail how PMT approaches the task of change propagation. My aim in this has not been to present PMT as the definitive change propagating 
model transformation approach; rather it has been to explore some of fundamentals of change propagation, to show workable design decisions for a resulting system, as well as to show how it works in practise. I therefore hope that PMT's approaches to transformation execution, target element identifiers, conformance operators, element removal and so on serve as a useful reference for future transformation users and language authors.

This research was funded by a grant from Tata Consultancy Services.

\section{REFERENCES}

[1] M. Alanen and I. Porres. Change propagation in a model-driven development tool. Presented at WiSME part of UML 2004, October 2004.

[2] S. M. Becker, T. Haase, and B. Westfechtel. Model-based a-posteriori integration of engineering tools for incremental development processes. SoSYM, 2004. To appear.

[3] J. Bézivin and S. Gérard. A preliminary identification of MDA components. In Generative Techniques in the context of Model Driven Architecture, Nov 2002.

[4] P. Braun and F. Marschall. Botl - the bidirectional object oriented transformation language. Technical Report TUM-I0307, Institut für Informatik der Technischen Universität München, May 2003.

[5] Compuware. OptimalJ, 2004. http://www.compuware.com/products/optimalj/.

[6] Compuware and Sun. XMOF queries, views and transformations on models using MOF, OCL and patterns, August 2003. OMG document ad/2003-08-07.

[7] K. Czarnecki and S. Helsen. Classification of model transformation approaches. In J. Bettin, G. van Emde Boas, A. Agrawal, E. Willink, and J. Bézivin, editors, Second Workshop on Generative Techniques in the context of Model Driven Architecture, October 2003.

[8] DSTC, IBM, and CBOP. MOF query / views / transformations first revised submission, August 2003. OMG document ad/2003-08-03.

[9] T. Gardner, C. Griffin, J. Koehler, and R. Hauser. Query / views / transformations submissions \& recommendations towards final standard, August 2003. OMG document ad/03-08-02.

[10] A. Gerber, M. Lawley, K. Raymond, J. Steel, and A. Wood. Transformation: The missing link of MDA. In A. Corradini, H. Ehrig, H.-J. Kreowski, and G. Rozenberg, editors, Graph Transformation: First International Conference, ICGT 2002, pages 90-105, October 2002. 
[11] J. Hunt and M. D. McIlroy. An algorithm for differential file comparison. Technical Report 41, Bell Laboratories Computing Lab, July 1976.

[12] S. Johann and A. Egyed. Instant and incremental transformation of models. September 2004.

[13] R. Jones and R. Lins. Garbage Collection: Algorithms for Automatic Dynamic Memory Management. Wiley, 1999.

[14] E. Kapsammer, T. Reiter, and W. Schwinger. Model-based tool integration state of the art and future perspectives. In Proc. CITSA 2006, July 2006.

[15] Object Management Group. Meta Object Facility (MOF) Specification, 2005. formal/05-05-05.

[16] QVT-Partners. First revised submission to QVT RFP, August 2003. OMG document ad/03-08-08.

[17] L. Tratt. The Converge programming language. Technical Report TR-05-01, Department of Computer Science, King's College London, February 2005.

[18] L. Tratt. Model transformations and tool integration. Journal of Software and Systems Modelling, 4(2):112-122, May 2005.

[19] L. Tratt. A change propagating model transformation language. Technical Report TR-06-XX, Department of Computer Science, King's College London, August 2006.

[20] L. Tratt. The MT model transformation language. In Proc. ACM Symposium on Applied Computing, pages 1296-1303, April 2006.

[21] L. Tratt and T. Clark. Issues surrounding model consistency and QVT. Technical Report TR-03-08, Department of Computer Science, King's College London, December 2003.

\section{ABOUT THE AUTHORS}

Laurence Tratt is a Senior Lecturer at Bournemouth University. See http://tratt.net/laurie/ for contact details. 